\title{
Synthesis and electrical properties of silver nanoplates for electronic applications
}

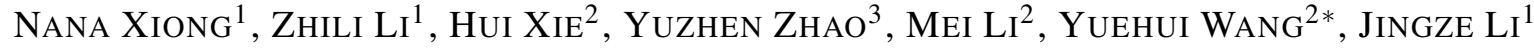 \\ ${ }^{1}$ State Key Laboratory of Electronic Thin Films and Integrated Devices, School of Microelectronics and Solid-State \\ Electronics, University of Electronic Science and Technology, Cheng Du, 610054, China \\ ${ }^{2}$ Department of Chemistry and Biology, University of Electronic Science and Technology of China Zhongshan Institute, \\ Zhong Shan, 528402, China \\ ${ }^{3}$ Department of Materials Science and Engineering, Tsinghua University, Beijing 100084
}

In this paper, silver nanoplates of 100 to $500 \mathrm{~nm}$ size were synthesized by reduction of silver nitrate with $\mathrm{N}, \mathrm{N}-$ dimethylformamide, using poly(vinylpyrolidone) as a surfactant and ferric chloride as a controlling agent, at 120 to $160{ }^{\circ} \mathrm{C}$ for 5 to 24 hours. The influence of the concentration of ferric chloride, the reaction temperature and reaction time on the morphology of the product has been investigated by transmission electron microscopy, scanning electron microscopy and UV-Vis spectroscopy. The results indicated that the products obtained at the low reaction temperature and short reaction time in the presence of $\mathrm{FeCl}_{3}$ in the reaction solution were in the form of silver nanoplates, whose morphology was mainly triangular and hexagonal. In addition, the size and thickness of the nanoplates increased with increasing of the $\mathrm{FeCl}_{3}$ concentration. At a high reaction temperature and long reaction time, the truncated triangle and hexagonal nanoplates were mainly produced. Furthermore, the sintering behavior of nanoplates was studied and the results showed that sintering of the silver nanoplates started at $180{ }^{\circ} \mathrm{C}$, and a typical sintering behavior was observed at higher temperatures. The incorporation of the silver nanoplates into the polymer matrix with micro-sized silver flakes led to an increase in the matrix resistivity in almost all cases, especially at high fractions and low curing temperatures. The curing temperature had an influence on the resistivity of the conductive adhesives filled with micro-sized silver flakes and silver nanoplates due to sintering of the silver nanoplates.

Keywords: silver nanoplates; solvothermal process; controlling agent; sintering behavior; electrical properties

(C) Wroclaw University of Technology.

\section{Introduction}

In recent years, a lot of research studies have been focused on the growth and properties of twodimensional silver $(\mathrm{Ag})$ nanomaterials due to their amazing ability to control optical properties and their promising applications in optics, photonics, electronics and biotechnology [1-18]. For example, silver nanostructures melting at a temperature well below the melting point of the bulk metal can be used as conductive fillers in electrically conductive adhesives (ECA) for application in microelectronic packaging [12, 13].

Therefore, it is very essential to develop a simple and effective preparation method of silver nanoparticles with controlled size and

*E-mail: wangzsedu@126.com shape [1-11, 14-22]. The methods of synthesis of silver nanostructures mainly include solvother$\mathrm{mal} /$ hydrothermal processes $[22,25]$ and, photoinduced [4], seed-mediated growth [3, 20, 21] as well as template $[1,18]$ methods. Investigations showed that the presence of controlling agents is an important factor to control the morphology of the silver nanostructures $[1-5,23,26]$. For example, Chen et al. $[23,26]$ reported that using of ferric chloride as a controlling agent is an effective strategy for producing silver nanowires/nanoplates. Im et al. [27] demonstrated that the introduction of hydrochloric acid to the conventional polyol method facilitates the formation of monodispersed silver nanocubes. Our group has done some works to synthesize silver nanostructures with different morphologies, such as nanospheres [19], nanocubes [28] and nanoplates [29]. However, the 
large-scale controllable synthesis of silver nanostructures has been a bottleneck for their using in commercial applications.

Nowadays, among silver nanostructures, silver nanoparticles and nanowires have been used as conductive fillers in ECAs. Compared with conventional microstructures, the higher surface area of nanostructures increases the contact area between the filler particles and decreases the percolation threshold, so the adhesives filled with silver nanostructures contain reduced silver filler content. This improves the viscosity and reduces internal stress of the resin mixtures, finally decreases the costs and makes possible to miniaturize the size of the electronic devices. However, it also inevitably increases the number of contact points and reduces the contact area among the conductive filler particles so that the resistivity of ECA is high $[12,13]$. Until now, academic reports concerning the effects of the silver nanostructures still present inconsistent conclusions [30-32]. Recently, the unique properties of silver nanostructures melting at a temperature well below the melting point of the bulk metal, increasingly arouse people's attention [3032]. Sintering of silver nanostructures, which fuse into each other and form metallurgical contacts, can improve the interfacial properties of conductive fillers and polymer matrices, reduce the contact resistance between the filler particles and increase the conductivity of ECA.

To the best of our knowledge, there has been no report on the effects of silver nanoplates as fillers on the electrical properties of ECA. In this paper, we report the fabrication of silver nanoplates via a solvothermal method by reducing $\mathrm{AgNO}_{3}$ with $\mathrm{N}, \mathrm{N}$-dimethylformamide (DMF), using poly(vinylpyrrolidone) (PVP) as a surfactant and ferric chloride $\left(\mathrm{FeCl}_{3}\right)$ as a controlling agent, followed by heat-treatment at 120 to $160{ }^{\circ} \mathrm{C}$ for 5 to 24 hours. It was found that the presence of $\mathrm{FeCl}_{3}$ had a significant effect on the synthesis of silver nanostructures with controllable morphologies. The influences of other reaction parameters, such as the concentration of $\mathrm{FeCl}_{3}$, the reaction temperature and reaction time, have also been investigated. Meanwhile, the sintering behavior of silver nanoplates was studied and characterization of silver nanoplates after heat treatment was performed. Further, the ECA were made by adding micro-sized silver flakes and silver nanoplates as hybrid fillers to the polymer composites consisting mainly of epoxy resin, curing agent and catalyst. Their properties were investigated in detail in terms of the fractions of silver nanoparticles in the total volume of silver filler, microstructure and the curing temperature.

\section{Experimental}

\subsection{Materials}

Silver nitrate $\left(\mathrm{AgNO}_{3}, \geqslant 99.8 \%\right)$ and poly(vinylpyrrolidone) (PVP, $\quad\left(\mathrm{C}_{6} \mathrm{H}_{9} \mathrm{NO}\right) n$, $\left.\mathrm{K} 30, \quad \mathrm{M}_{w}=40000\right)$ were purchased from Sinopharm Group Chemical Reagent Co., Ltd., and $\mathrm{N}, \mathrm{N}$-dimethylformamide (DMF, $\mathrm{HCON}\left(\mathrm{CH}_{3}\right)_{2}$, $\geqslant 99.5 \%$ ) was purchased from Shanghai Chemical Reagent Co., Ltd., Ferric chloride $\left(\mathrm{FeCl}_{3} \cdot 6 \mathrm{H}_{2} \mathrm{O}, \geqslant 99.0 \%\right)$ and absolute ethanol $\left(\mathrm{CH}_{3} \mathrm{CH}_{2} \mathrm{OH}, \geqslant 99.7 \%\right)$ were purchased from Tianjin Yongda Chemical Co., Ltd., Diglycidyl ether of bisphenol A (R-128) was purchased from Guangzhou Hongchang Co., Ltd. 4-methylhexahydrophthalic anhydride (MHHPA) and 2-ethyl-4-methylimidazole (2E4MZ) were supplied by Guangtuo Chemical Co., Ltd. Silver flakes (SF1023K, $\mathrm{D}_{90}<5 \mu \mathrm{m}$ ) were purchased from Guangdong Fenghua Advanced Technology Group Co., Ltd. All chemicals were used without further purification.

\subsection{Synthesis of silver nanoplates and ECA preparation}

In a typical experimental process, $0.68 \mathrm{~g}$ PVP was dissolved into $40 \mathrm{~mL}$ of $100 \mu \mathrm{mol} \cdot \mathrm{L}^{-1} \mathrm{FeCl}_{3}$ DMF solution. $0.68 \mathrm{~g} \mathrm{AgNO}_{3}$ was dissolved into $40 \mathrm{~mL}$ DMF solution, and was then rapidly injected into the vigorously magnetically stirred DMF solution with PVP and $\mathrm{FeCl}_{3}$ in a minute. The solution turned brown immediately, and it was further stirred for another $15 \mathrm{~min}$ and transferred into a $100 \mathrm{~mL}$ Teflon-lined autoclave tube. The tube was sealed and maintained at $160{ }^{\circ} \mathrm{C}$ for $6 \mathrm{~h}$, 
followed by natural cooling to room temperature. The color of the reaction solution was gray and deepened with increasing of $\mathrm{FeCl}_{3}$ concentration. Silver nanostructures were purified by centrifuging at $5000 \mathrm{rpm}$ for $15 \mathrm{~min}$ in the presence of acetone. The purified silver nanostructures were dispersed in DI water for further characterization.

R-128, MHHPA and 2E4MZ with a weight ratio of 1:0.85:0.05 were put into a small beaker, and sonicated for $30 \mathrm{~min}$. Then, the micro-sized silver flakes were incorporated into the polymer matrix by sonication for another $30 \mathrm{~min}$ to make the filler uniformly dispersed in the mixture, and the silver nanoplates were also incorporated into the polymer matrix after sonication for more than 60 min to disperse the conductive fillers. Two strips of polyimide tape were applied onto a pre-cleaned glass slide with a gap width of $1 \mathrm{~cm}$. The formulated composite was injected into the space between the two strips. The polyimide tapes were removed before curing.

\subsection{Characterization}

All transmission electron microscope (TEM) images were taken using a JEOL 200CX TEM operated at $200 \mathrm{kV}$. Scanning electron microscopy (SEM) images were taken on a JSM-6460. X-ray diffraction (XRD) patterns of the nanoplates were recorded by a Rigaku D8-Discover diffractometer using $\mathrm{CuK} \alpha$ radiation $(\lambda=1.5418 \AA)$ and a graphite monochromator, at a scanning rate of 0.02 degree per second in $2 \theta$ range of 20 to $85^{\circ}$. The UV-Vis absorption spectra were obtained using a 760CRT spectrophotometer with quartz cuvettes at room temperature. The resistivity of the ECA was measured using a DMR-1C four-point probe meter (Nanjing Daming instrument Co., Ltd.). The resistivity, $\rho$, was calculated using the following equation:

$$
\rho=R_{L} \omega
$$

where $R_{L}$ and $\omega$ are resistance per square and thickness of the sample, respectively. The thickness of the cured samples was measured by a micrometer gauge. The average sheet resistance and the thickness of the sample were estimated from the measurements at six points chosen on the sample surface.

\section{Results and discussion}

\subsection{Characterization of silver nanoplates}

To clarify the role of $\mathrm{FeCl}_{3}$ in the growth process, we conducted the experiments using the procedure similar to that described in section 2.2, except that different $\mathrm{FeCl}_{3}$ concentrations were used. Fig. 1 shows the SEM images of silver nanostructures prepared with $0,0.05,0.1,0.3$ and 0.4 $\mathrm{mmol} \cdot \mathrm{L}^{-1} \mathrm{FeCl}_{3}$, respectively. In the absence of $\mathrm{FeCl}_{3}$, there are mixed nanostructures of silver nanoplates, nanoparticles and a few nanorods (Fig. 1a). When $\mathrm{FeCl}_{3}$ was added into the reaction solution, irregular nanoplates, including triangular, quadrilateral, pentagon, hexagonal structures, shown in Fig. 1b to 1e, were obtained. With increasing of $\mathrm{FeCl}_{3}$ concentration from 0.05 to 0.4 $\mathrm{mmol} \cdot \mathrm{L}^{-1}$, the number of hexagonal nanoplates and their size and thickness increased. The range of sizes of as-prepared nanostructures is 100 to $500 \mathrm{~nm}$. Results of the research show that the introduction of $\mathrm{FeCl}_{3}$ to the reaction solution plays an important role in the formation of silver nanoplates. It is mainly because in the initial stage, $\mathrm{AgCl}$ colloids are formed that control the free $\mathrm{Ag}^{+}$ions concentration in the DMF solution, which promotes the growth of silver nanoplates $[23,26]$.

In order to understand the formation of silver nanoplates, we measured the UV-Vis absorption spectra of the silver nanostructures obtained at different concentrations of $\mathrm{FeCl}_{3}$, shown in Fig. 1. The appearance of the broad and intense peak at $\sim 420 \mathrm{~nm}$ (Fig. 2 curve a) indicates the formation of silver nanoparticles [17, 19]. In addition, the absorption peak at $\sim 355 \mathrm{~nm}$ and absorption band in the range of 500 to $800 \mathrm{~nm}$ indicate the presence of mixed silver nanoplates, nanoparticles and nanorods with different sizes. For $\mathrm{FeCl}_{3}$ concentration of $0.05 \mathrm{mmol} \cdot \mathrm{L}^{-1}$ (Fig. 2 curve b), the peak is considerably blue-shifted from $420 \mathrm{~nm}$ to $385 \mathrm{~nm}$, which is accompanied by a weaker absorption intensity at $355 \mathrm{~nm}$ and a very strong absorption band in the range of 420 to $800 \mathrm{~nm}$ 

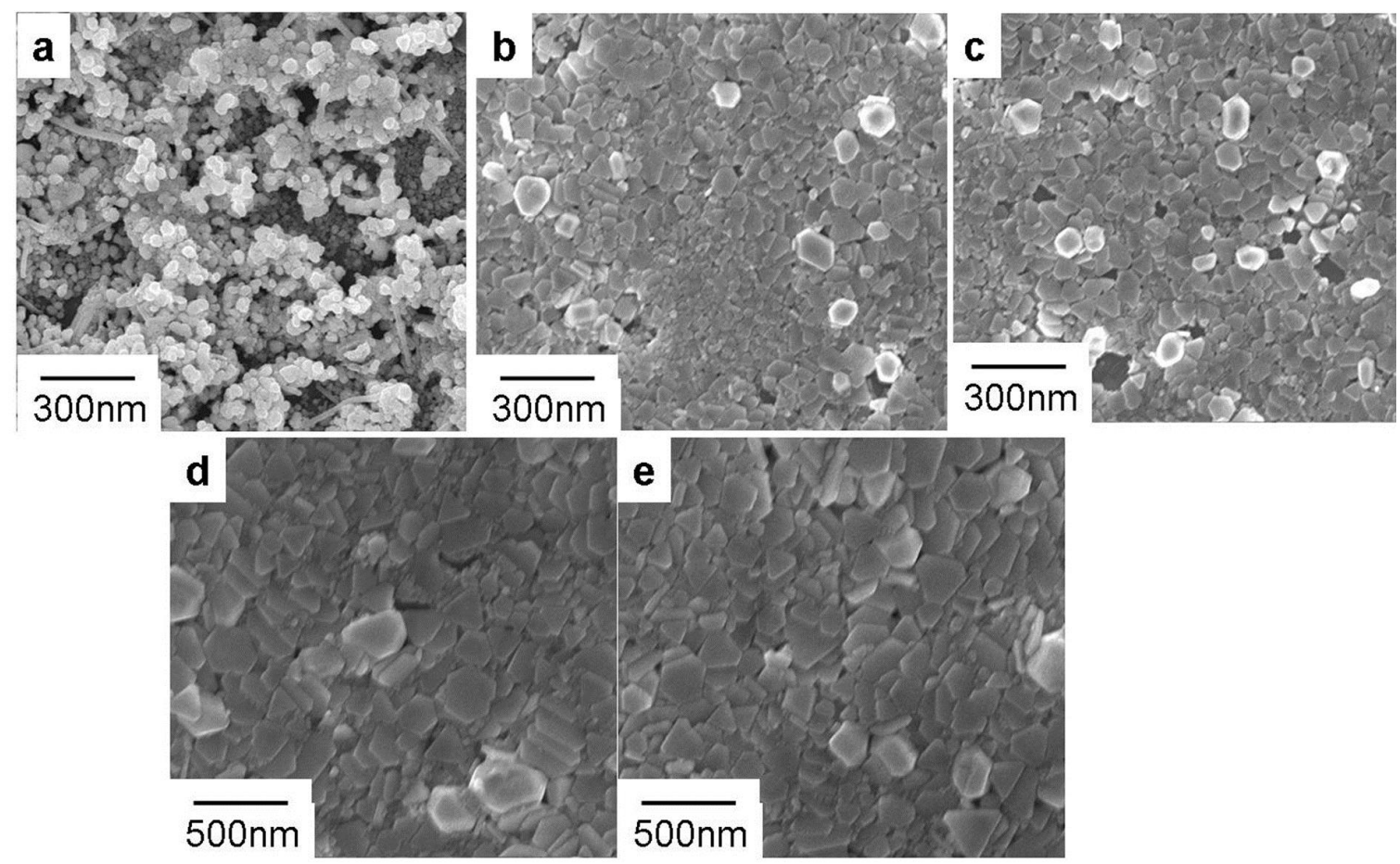

Fig. 1. SEM images of silver nanostructures prepared with different $\mathrm{FeCl}_{3}$ concentrations: (a) 0, (b) 0.05, (c) 0.1, (d) 0.3 and (e) $0.4 \mathrm{mmol} \cdot \mathrm{L}^{-1}$.

because of the appearance of silver nanoplates. Based on theoretical calculations by Jin et al. [4], the $355 \mathrm{~nm}$ peak can be related to the out-ofplane quadrupole plasmon resonance, whereas the $390 \mathrm{~nm}$ peak results from the in-plane quadrupole plasmon resonance $[33,34]$. It is worth noting that the peak at $335 \mathrm{~nm}$ has not changed in all the experimental conditions. This result indicates that the out-of-plane quadrupole plasmon resonance is not affected by the morphology and size of silver nanoplates. With the increase of $\mathrm{FeCl}_{3}$ concentration from 0.05 to $0.4 \mathrm{mmol} \cdot \mathrm{L}^{-1}$, the absorption peak has red-shifted from $385 \mathrm{~nm}$ to $405 \mathrm{~nm}$, which indicates an increase in the size of nanoplates [36]. The result of the analysis of UV-Vis absorption spectra is consistent with that of the SEM images (Fig. 1).

The effects of the reaction temperature and reaction time on the morphology of silver nanostructures were investigated. Fig. 3 shows the UVVis absorption spectra of silver nanostructures prepared using a procedure similar to that described in section 2.2, at different reaction times. It is clear

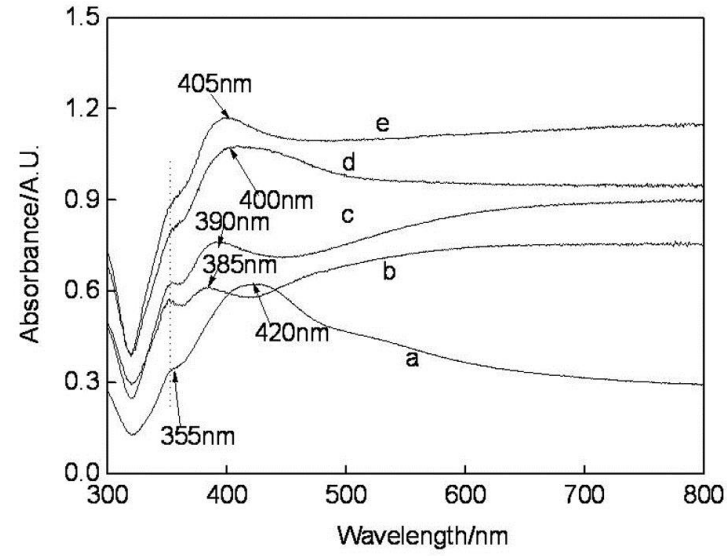

Fig. 2. UV-Vis absorption spectra of silver nanostructures prepared with different $\mathrm{FeCl}_{3}$ concentrations: (a) 0 , (b) 0.05 , (c) 0.1 , (d) 0.3 and (e) $0.4 \mathrm{mmol} \cdot \mathrm{L}^{-1}$.

that silver nanoplates were obtained after $5 \mathrm{~h}$, and the extension of reaction time caused that the peak blue-shifted from $427 \mathrm{~nm}$ to $392 \mathrm{~nm}$, which indicates that the size of the structures became smaller and they gained hexagonal shapes with truncated angles [36]. 


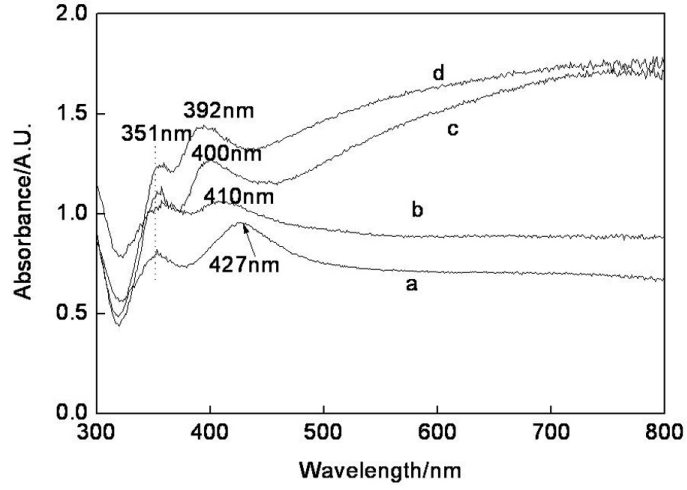

Fig. 3. UV-Vis absorption spectra of silver nanostructures prepared during different reaction times: (a) $5 \mathrm{~h}$, (b) $10 \mathrm{~h}$, (c) $15 \mathrm{~h}$ and (d) $24 \mathrm{~h}$.

Fig. 4 shows TEM images of silver nanoplates prepared for 5, 10, 15 and $24 \mathrm{~h}$, respectively. As can be seen from Fig. 4, after 5 h, triangular and hexagonal silver nanoplates were obtained (Fig. 4a), and after $15 \mathrm{~h}$, the structures changed into the truncated triangle and hexagonal nanoplates (Fig. 4d). Fig. 5 shows the UV-Vis absorption spectra of silver nanostructures prepared with $0.1 \mathrm{mmol} \cdot \mathrm{L}^{-1} \mathrm{FeCl}_{3}$ at different reaction temperatures for $6 \mathrm{~h}$. The curves $\mathrm{a}, \mathrm{b}$ and $\mathrm{c}$ were taken for the samples prepared at $120{ }^{\circ} \mathrm{C}, 130{ }^{\circ} \mathrm{C}$ and $150{ }^{\circ} \mathrm{C}$, respectively. As can be seen from Fig. 5, the UV-Vis absorption spectra have characteristics typical of silver nanoplates. The main difference is that for the sample obtained at the reaction temperature of $150{ }^{\circ} \mathrm{C}$ there is an intense absorption in the long wavelength band. It indicates that not only the size and thickness of the silver nanoplates became larger but also their morphologies changed into truncated triangle and hexagonal nanoplates. The above phenomena suggest that the reaction temperature and the reaction time have an important effect on the morphology of silver nanoplates.

Fig. 6 shows a typical powder XRD pattern of the as-prepared silver nanoplates. The intensive diffraction peak located at $2 \theta=38^{\circ}$, comes form the (111) plane of fcc silver (JCPDS No. 4-0783). All these observations reveal that the nanoplates basal plane is the (111) plane, which is consistent with previous studies on silver nanocrystals bounded by atomically flat surfaces. However, no

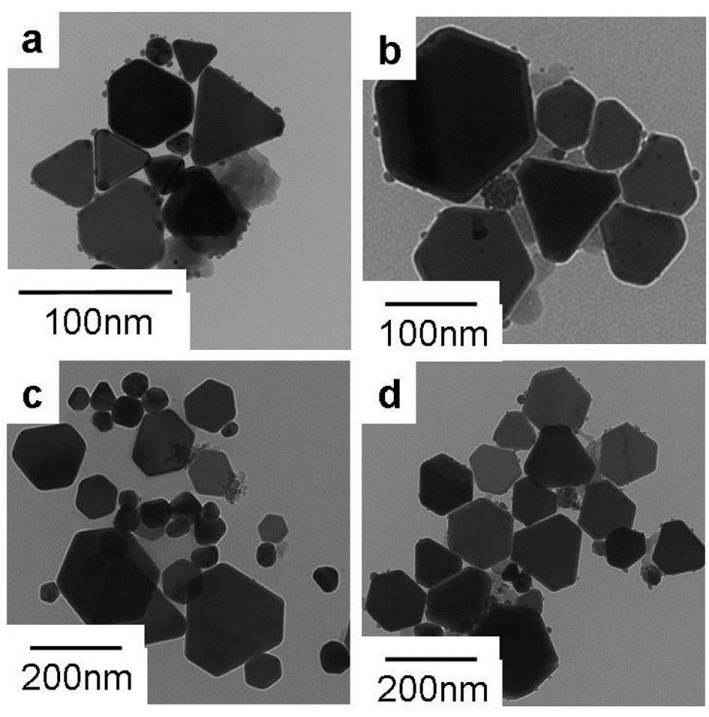

Fig. 4. TEM images of silver nanostructures prepared during different reaction times: (a) $5 \mathrm{~h}$, (b) $10 \mathrm{~h}$, (c) $15 \mathrm{~h}$ and (d) $24 \mathrm{~h}$.

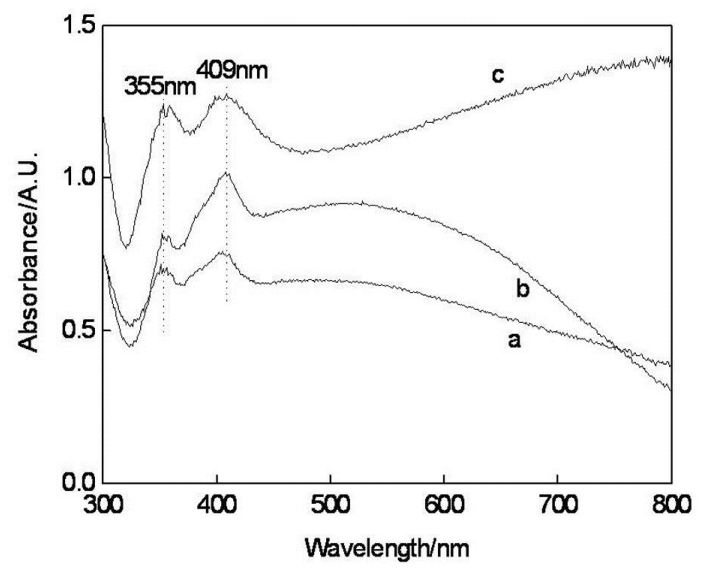

Fig. 5. UV-Vis absorption spectra of silver nanostructures prepared at different reaction temperatures for $6 \mathrm{~h}$ : (a) $120{ }^{\circ} \mathrm{C}$, (b) $130{ }^{\circ} \mathrm{C}$ and (c) $150{ }^{\circ} \mathrm{C}$.

diffraction peaks of $\mathrm{AgCl}$ colloids, which formed in the initial stage, can be observed in Fig. 6. This result indicates that the introduction of $\mathrm{FeCl}_{3}$ to the reaction solution has a limited effect on the purity of silver nanoplates. The $\mathrm{FeCl}_{3}$ added into the reaction solution caused that the $\mathrm{AgCl}$ colloids were formed in the initial stage, which resulted in decreasing free $\mathrm{Ag}^{+}$ions during the initial formation of silver seeds and slow releasing of $\mathrm{Ag}^{+}$ions to 
the solution during the subsequent reaction. This led to the formation of silver nanoplates.

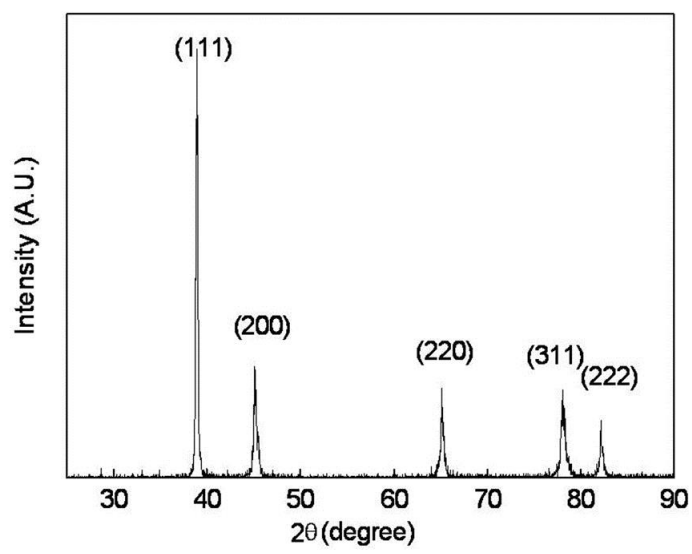

Fig. 6. XRD pattern of the as-prepared silver nanoplates.

\subsection{Sintering behavior of silver nanoplates}

Fig. 7 shows the SEM images of the silver nanoplates sintered on glass substrates at 180, 200, 250 and $300{ }^{\circ} \mathrm{C}$ for $30 \mathrm{~min}$, respectively. It can be seen that the silver nanoplates growth and shape accommodation took place after sintering. The sintering started at $180{ }^{\circ} \mathrm{C}$, as shown in Fig. $7 \mathrm{~b}$. At $250{ }^{\circ} \mathrm{C}$, silver nanoplates were almost completely sintered and formed a porous network. (Fig. 7c). It seems that the nanoplates may assist to decrease the melting point. At $300{ }^{\circ} \mathrm{C}$, the silver nanoplates formed large chunks dispersed in the porous network.

\subsection{ECA filled with micro-sized silver flakes and nanoparticles}

Fig. 8 shows the resistivity of the ECA filled with micro-sized silver flakes and different fractions of silver nanoplates in $75 \mathrm{wt} \%$ of silver filler after curing at $180,200,250$, and $300{ }^{\circ} \mathrm{C}$ for $60 \mathrm{~min}$. It can be seen that the resistivities of the polymer matrixes doped with silver nanoplates increase in all cases, especially in the case of the high fraction of the nanoplates and low curing temperature. When $30 \mathrm{wt}$ \% silver nanoplates of the total of silver filler were doped into the ECA cured
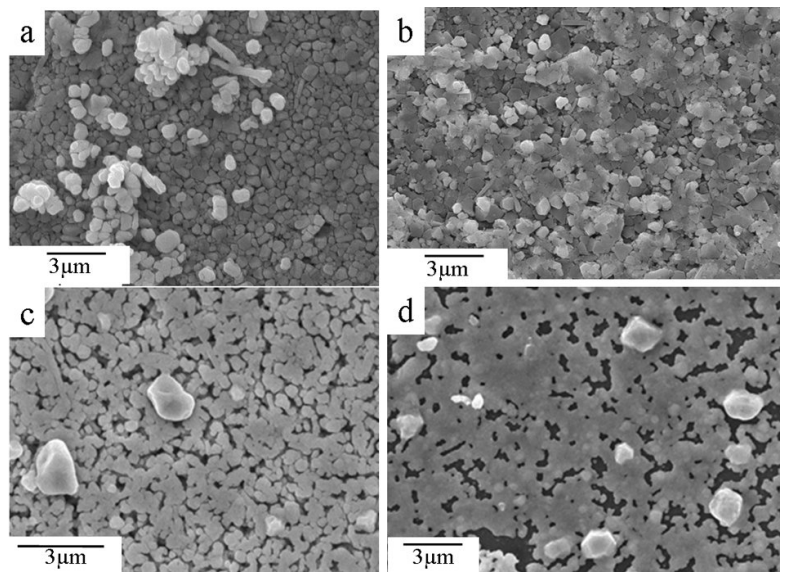

Fig. 7. SEM images of the silver nanoplates sintered on glass substrate at different temperatures for 30 min: (a) $150{ }^{\circ} \mathrm{C}$, (b) $180{ }^{\circ} \mathrm{C}$, (c) $250{ }^{\circ} \mathrm{C}$, (d) $300{ }^{\circ} \mathrm{C}$.

at $180{ }^{\circ} \mathrm{C}, 200{ }^{\circ} \mathrm{C}$ and $250{ }^{\circ} \mathrm{C}$, the resistivity increased 2700 times, 2560 times and 5.02 times, respectively. In literature it was implied that doping with silver nanostructures inevitably increases the contact resistance. However, the nanostructures, due to their small size, may enter into the gaps between unattached silver flakes, built a new network, and, in this way, reduce slightly the ESA resistivity [30-32]. It is clear that in our experiment the role of the silver nanoplates is mainly to increase the contact resistance. In the system of ECA filled with the micro-sized silver flakes and nanoplates as hybrid fillers, with the increase of the content of silver nanoplates in the total filler, the content of micro-sized silver flakes gradually decreases, so that the conductive network established by the silver flakes obviously decreases and the contact resistance increases. The experimental results indicate that the improvement of the electrical conductivity of the ECA depends on the synergy of silver flakes and nanoplates. In other words, it means that silver nanoplates mixed with silver flakes only play limited and auxiliary role in conductive networks. In addition, it can be seen from Fig. 8, that the resistivity of the ECA reduces with increasing of the curing temperature, especially at 250 and $300{ }^{\circ} \mathrm{C}$ when the nanoplates start fusing and sintering. It means that the temperature affects the conductivity of the ECA doped with silver nanoplates. 


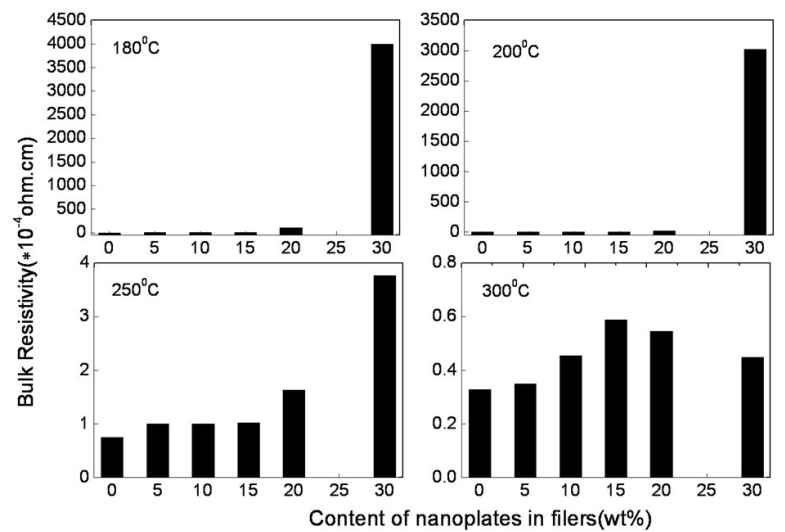

Fig. 8. Resistivity of the ECA filled with micro-sized silver flakes and different fractions of silver nanoplates in $75 \mathrm{wt} . \%$ of silver filler after curing at $180{ }^{\circ} \mathrm{C}, 200{ }^{\circ} \mathrm{C}, 250{ }^{\circ} \mathrm{C}$, and $300{ }^{\circ} \mathrm{C}$ for $60 \mathrm{~min}$.

Fig. 9 shows SEM images of the ECA filled with micro-sized silver flakes and $5 \%, 10 \%, 15 \%$, $20 \%$ and $30 \%$ silver nanoplates in $75 \mathrm{wt} . \%$ of silver filler after curing at $180{ }^{\circ} \mathrm{C}$ for $60 \mathrm{~min}$. As can be seen from Fig. 9, the silver nanoplates adsorb on the surface of the silver flakes, and with increasing of the content of silver nanoplates, more and more silver nanoplates adsorb and cover the surfaces of the silver flakes. It also explains the reasons why the resistivity increases with increasing of the content of silver nanoplates. It is worth pointing out that the distance between the silver nanoplates is large so that the tunneling current can hardly happen. Based on the results presented above, it can be stated that incorporation of the silver nanoplates into the polymer matrix deteriorated the conductivity, so we think that the effective dispersion of fillers in the polymer matrix to establish more conductive paths is the key factor to obtain the high electrical conductivity.

Fig. 10 shows SEM images of the ECA filled with micro-sized silver flakes and 10 and $30 \%$ silver nanoplates (Fig. 10a and 10b) in $75 \mathrm{wt} . \%$ of silver filler cured at $200{ }^{\circ} \mathrm{C}, 250{ }^{\circ} \mathrm{C}$ and $300{ }^{\circ} \mathrm{C}$ for $60 \mathrm{~min}$, respectively. Sintering of the silver filler is observed at $250{ }^{\circ} \mathrm{C}$. At $300{ }^{\circ} \mathrm{C}$, the silver nanoplates and silver flakes form sintered blocks whose morphology is similar to the morphology typical of sintered ceramic powders. However, the morphologies of the sintered fillers cured at $300{ }^{\circ} \mathrm{C}$ almost do not depend on the content of silver nanoplates. It also explains the reason why the differences in conductivities were so little when the ECA were cured at $300{ }^{\circ} \mathrm{C}$.

In order to further analyze the effect of silver nanoplates on the conductivity of ECA, we also studied the resistivity of the ECA filled with micro-sized silver flakes and different fractions of silver nanoplates in $65 \mathrm{wt} \%$ of silver filler after curing at 180,250 , and $300{ }^{\circ} \mathrm{C}$ for $60 \mathrm{~min}$, as shown in Fig. 11. Obviously, the incorporation of the nanoplates resulted in increasing of the resistivity, except the sample cured at $300{ }^{\circ} \mathrm{C}$. It is likely that the fusing and sintering of silver nanoplates at $300{ }^{\circ} \mathrm{C}$ helped to fill the gaps between silver flakes and build the conductive network which reduced the resistivity of the composite.

\section{Conclusions}

Silver nanoplates were synthesized through reducing of silver nitrate with $\mathrm{N}, \mathrm{N}-$ dimethylformamide, using poly(vinylprrolidone) (PVP) as a surfactant and ferric chloride as a controlling agent, at 120 to $160{ }^{\circ} \mathrm{C}$ for 5 to 24 hours. The influence of the concentration of ferric chloride, the reaction temperature and reaction time on the morphologies of the products has been investigated by transmission electron microscopy, scanning electron microscopy and UV-Vis spectroscopy. The results indicated that the products obtained in the presence of $\mathrm{FeCl}_{3}$ in the reaction solution were in the form of silver nanoplates, and the morphologies of the silver nanoplates prepared at low reaction temperatures and the short reaction times were mainly triangular and hexagonal. In addition, the size and thickness of the structures increased with increasing of the $\mathrm{FeCl}_{3}$ concentration. The truncated triangle and hexagonal nanoplates were the main products obtained at high reaction temperatures and long reaction times. In addition, the sintering behavior of nanoplates was studied and the results showed that the silver nanoplates began sintering at $180{ }^{\circ} \mathrm{C}$, and a typical sintering behavior was observed at higher temperatures. The incorporation of the 


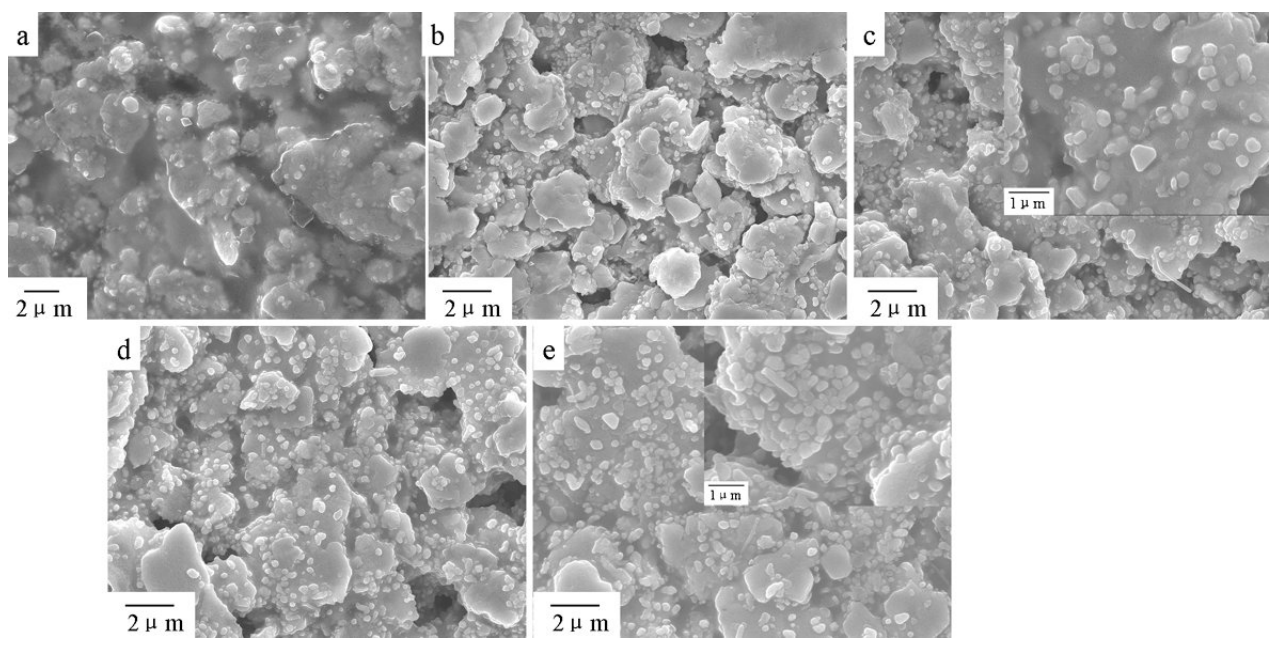

Fig. 9. SEM images of ECA with micro-sized silver flakes and (a) $5 \%$, (b) $10 \%$, (c) $15 \%$, (d) $20 \%$ and (e) $30 \%$ of silver nanoplates in $75 \mathrm{wt} . \%$ silver filler after curing at $180{ }^{\circ} \mathrm{C}$ for $60 \mathrm{~min}$. The insets show magnified images.

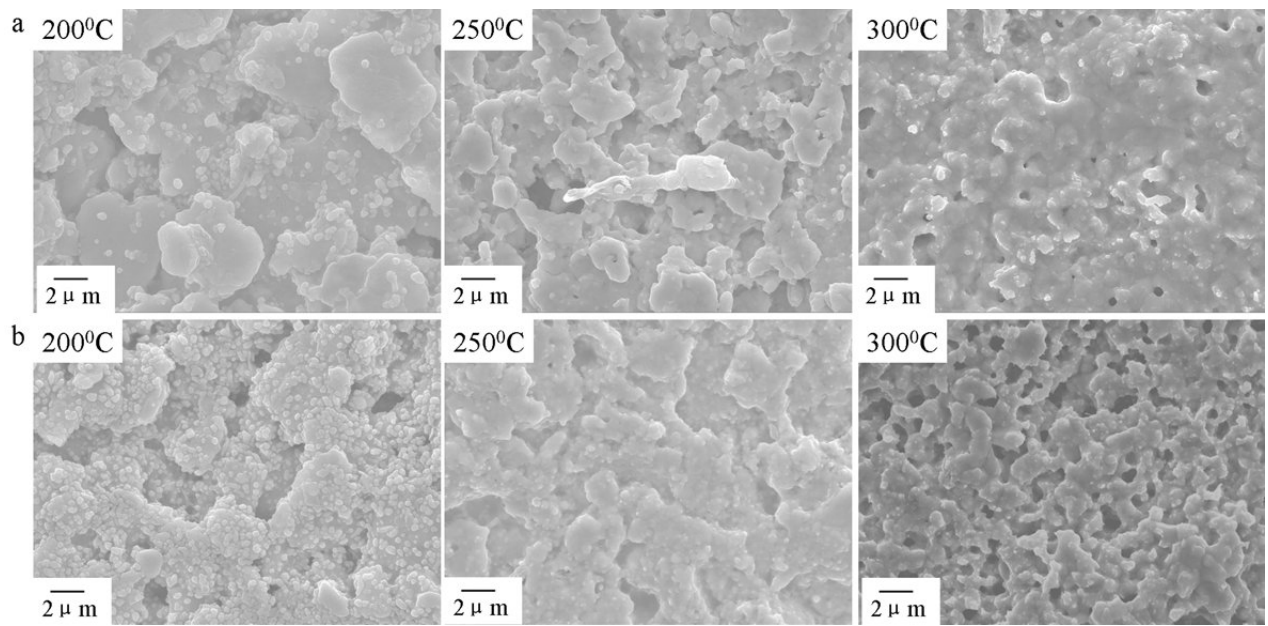

Fig. 10. SEM images of the ECAs filled with micro-sized silver flakes and (a) $10 \%$, (b) $30 \%$ of silver nanoplates in 75 wt. $\%$ silver filler cured at $200{ }^{\circ} \mathrm{C}, 250{ }^{\circ} \mathrm{C}$ and $300{ }^{\circ} \mathrm{C}$ for $60 \mathrm{~min}$.

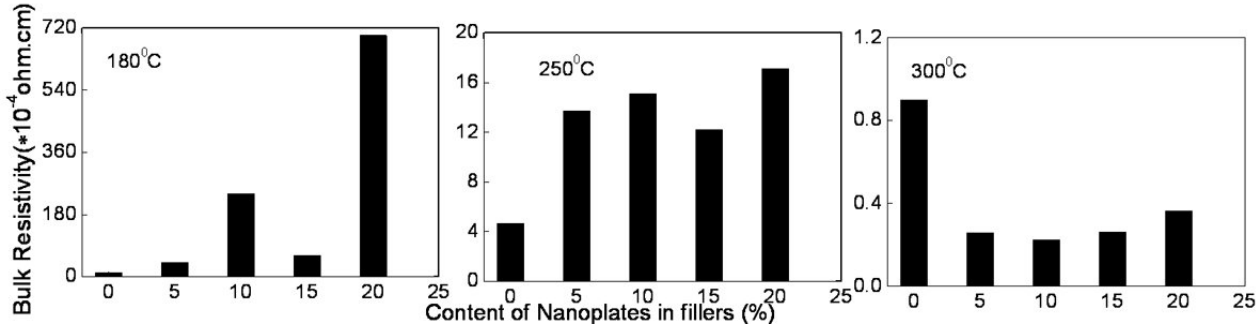

Fig. 11. Resistivity of the ECA filled with micro-sized silver flakes and different fractions of silver nanoplates in 65 wt. $\%$ of silver filler after curing at $180{ }^{\circ} \mathrm{C}, 250{ }^{\circ} \mathrm{C}$, and $300{ }^{\circ} \mathrm{C}$ for $60 \mathrm{~min}$. 
silver nanoplates into the polymer matrix with micro-sized silver flakes led to the increase in the resistivity in almost all cases, especially at the high fractions and low curing temperatures. The curing temperature had an influence on the resistivity of the ECA filled with micro-sized silver flakes and silver nanoplates due to sintering of the silver nanoplates.

\section{Acknowledgements}

This work was financially supported by National Science Foundation of China under grants of (61302044, 51302145) and Zhongshan Science and Technology Projects (20123A319, 2014A2FC312) and State Key Laboratory of New Ceramic and Fine Processing (Tsinghua University) and State Key Laboratory of Electronic State Key Laboratory of Electronic Thin Film and Integrate (Zhongshan).

\section{References}

[1] Sun Y., Xia Y., Adv. Mater., 15 (9) (2003), 695.

[2] Chen S., Carroll D., J. Phys. Chem. B, 108 (2004), 5500.

[3] Wiley B.J., Wang Z., Wei J., Yin Y., Cobden D.H., XIA Y., Nano Lett., 6 (10) (2006), 2273.

[4] Jin R., CaO Y., MiRkin C.A., Kelly K.L., SchatZ G.C., Zheng J.G., Science, 294 (2001), 1901.

[5] Kelly K.L., Coronado E., Zhao L.L., Schatz G.C., J. Phys. Chem. B, 107 (3) (2003), 668.

[6] Zhang J., Li S., Wu J., Schatz G.C., Mirkin C.A., Angew. Chem. Int. Edit., 48 (42) (2009), 7787.

[7] Banholzer M.J., Osberg K.D., Li S., MangelSON B.F., SCHATZ G.C., MiRKIN C.A., ACS Nano, 284 (9) (2010), 5446.

[8] Tang B., Xu S., Hou X., Li J., Sun L., XU W., WANG X., ACS Appl. Mater. Inter., 5 (3) (2013), 646.

[9] KIM Y.K., MIN D.H., RSC Adv., 4 (14) (2014), 6950.

[10] Yang Y., Zhong X.L., Zhang Q., Blackstad L.G., FU Z.W., LI Z.Y., QIN D., Small, 10 (7) (2014), 1430.

[11] Li Z., Meng G., Liang T., Zhang Z., Zhu X., Appl. Surf. Sci., 264 (2013), 383.

[12] Zhang R.W., Moon K.S., Lin W., Wong C.P., J. Mater. Chem., 20 (2010), 2018.

[13] Zhang Z.X., Chen X.Y., Xiao F., Polym. Advan. Technol., 25 (2011), 1465.

[14] LaI Y., PAN W., Zhang D., Zhan J., Nanoscale, 3 (5) (2011), 2134.
[15] Zeng J., TAO J., Li W., GRant J., WAng P., ZhU Y., XIA Y., Chem.-Asian J., 6 (2) (2011), 376.

[16] CaO Z.W., Fu H.B., Kang L., Huang L.W., ZHaI T.Y., Ma Y., YAO J.N., FU H.B., J. Mater. Chem., 18 (23) (2006), 2673.

[17] Xiong Y., SiekKinen A.R., Wang J., J. Mater. Chem., 17 (25) (2007), 2600.

[18] Washio Y., Xiong Y., Yin Y., Xia Y., Adv. Mater., 18 (2006), 1745.

[19] Wang Y.H., Zhang Q., Wang T., Zhou J., Chinese J. Inorg. Chem., 26 (3) (2010), 365.

[20] Li N., Zhang Q., Quinlivan S., Goebl J., Gan Y., YIN Y., ChemPhysChem, 13 (2012), 2526.

[21] PARK J., Yoon D.-Y., KIM Y., Korean J. Chem. Eng., 26 (1) (2009), 258.

[22] Roh J., PArk E.-J., PArk K., Yi J., Kim Y., J. Chem. Eng., 27 (6) (2010), 1897.

[23] Chen D., Qiao X., QIU X., Chen J., JiAng R., J. Mater. Sci.-Mater. El., 22 (2011), 6.

[24] Zhang W.C., Wu X.L., Chen H.T., Gao Y.J., ZhU J., Huang G.S., Chu P.K., Acta Mater, 56 (1) (2008), 2508.

[25] Zeng J., Xia X., Rycnega M., Henneghan P., XIA Y., Angew. Chem. Int. Edit., 50 (1) (2011), 244.

[26] Chen D., Zhu X., Zhu G., Qiao X., Chen J., J. Mater. Sci.-Mater. El., 23 (2012), 625.

[27] Im S.H., LeE Y.T., Wiley B., XiA Y., Angew. Chem. Int. Edit., 44 (2005), 2154.

[28] Wang H., Zhang Q., Wang T., Zhou J., Int. Mater. Rev., 22 (3) (2008), 144.

[29] Wang Y.H., Wang T., Zhou J., Chinese J. Inorg. Chem., 23 (8) (2007), 1485.

[30] Li Y., Moon K.S., Wong C.P., J. Electron. Mater., 99 (2006), 1573.

[31] Cui H.W., Li D.S., FAn Q., Electron. Mater. Lett., 9 (2013), 299.

[32] Lu D.D., Li Y.G., Wong C.P., J. Adhes. Sci. Technol., 22 (2008), 815.

[33] Jin R.C., CaO Y.C., Hao E.C., Metraux G.S., Schatz G.C., Mir Kin C.A., Nature, 425 92003), 487.

[34] Sherry L.J., Jin R.C.,Mirkin C.A., SChatz G.C., VAN DUYNe R.P., Nano Lett., 6 (9) (2006), 2060.

[35] Hao E., Schatz G., Hupp J., J. Fluoresc., 14 (4) (2004), 331.

[36] Pastoriza-Santos I., Liz-MarzÁn L.M., Nano Lett., 2 (2002), 903. 